\title{
FEDSM-ICNMM2010-30214
}

\section{MEASUREMENT OF INTERFACIAL AREA PRODUCTION AND PERMEABILITY WITHIN POROUS MEDIA}

\author{
Dustin Crandall \\ URS/Washington Division \\ National Energy Technology Laboratory \\ Morgantown, WV, USA \\ Dustin.Crandall@ur.netl.doe.gov
}

\author{
Goodarz Ahmadi \\ Mechanical and Aeronautical Engineering \\ Clarkson University \\ Potsdam, NY, USA
}

\author{
Duane H Smith \\ National Energy Technology Laboratory \\ Morgantown, WV, USA
}

\begin{abstract}
An understanding of the pore-level interactions that affect multi-phase flow in porous media is important in many subsurface engineering applications, including enhanced oil recovery, remediation of dense non-aqueous liquid contaminated sites, and geologic $\mathrm{CO}_{2}$ sequestration. Standard models of two-phase flow in porous media have been shown to have several shortcomings, which might partially be overcome using a recently developed model based on thermodynamic principles that includes interfacial area as an additional parameter. A few static experimental studies have been previously performed, which allowed the determination of static parameters of the model, but no information exists concerning the interfacial area dynamic parameters. A new experimental porous flow cell that was constructed using stereolithography for two-phase gas-liquid flow studies was used in conjunction with an in-house analysis code to provide information on dynamic evolution of both fluid phases and gasliquid interfaces. In this paper, we give a brief introduction to the new generalized model of two-phase flow model and describe how the stereolithography flow cell experimental setup was used to obtain the dynamic parameters for the interfacial area numerical model. In particular, the methods used to determine the interfacial area permeability and production terms are shown.
\end{abstract}

\section{INTRODUCTION}

A number of engineering problems require knowledge of flow in porous media, including applications in the environment, biology, industry, oil \& gas recovery, and geologic sequestration of carbon dioxide. These applications range from the movement of fluids and particulates in the subsurface, to brain and liver cancer treatment, to processes occurring during paper manufacturing and within fuel cells. Modeling of these systems is usually performed with a standard set of equations based upon Darcy's Law (Darcy, 1856); a century and a half old phenomenological relationship describing the flow of a single fluid through a homogenous porous domain. For systems consisting of two fluids moving within a porous medium, an extended form of Darcy's law to determine fluid phase velocities and saturations has been used for decades (Helmig, 1997). From these velocities and saturations functional relationships to determine the capillary pressure $\left(p_{c}\right)$ and relative permeabilities $\left(k_{r}\right)$ of the system are typically used. This method is deficient with respect to physics in that it completely neglects the role and presence of fluidfluid interfaces and leads to hysteretic relationships. Interfaces between fluids are not only crucial quantities for interfacedependent processes, such as mass transfer between phases, but there is a growing amount of experimental evidence that shows a relationship between $\mathrm{p}_{\mathrm{c}}$, interfacial area, and saturation $(S)$ accounts for the observed hysteresis in the $k_{r}-S$ relationship by collapsing the curves upon a single surface (Culligan et al. 2004, Brusseau et al. 2006, Chen and Kibbey 2006, PyrakNolte et al. 2008).

Alternative theories of multi-phase flow and transport in porous media have been developed. Among these are theories based on rational thermodynamics by Hassanizadeh and Gray $(1980,1990)$ and theories based on thermodynamically constrained averaging theory by Gray and Miller (2005, Miller 
and Gray 2005). In addition, there is an approach which separates balance equations for percolating and non-percolating part of each phase (Hilfer and Doster, 2009). In this work we use the approach of Hassanizadeh and Gray (1980, 1990), which is based on thermodynamic principles and is more physically-based than the standard modified Darcy's Law model. This new approach not only includes balance equations for the bulk phases, but also an interfacial balance equation. Furthermore, in this theory $p_{c}$ is not a function of $S$ only, but also depends on specific interfacial area $\left(a_{w n}\right)$. A modeling approach using this theory with idealized parameters was put into practice by Niessner and Hassanizadeh (2008) that includes balance equations for two bulk fluid phases and the fluid-fluid interface, a functional relationship between $a_{w n}, p_{c}$, $S$, and a production rate term of interfacial area $\left(E_{w n}\right)$.

In order to properly use this new model the necessary parameters must be obtained. While many static tests have been performed to calculate relations between $a_{w n}, p_{c}$, and $S$, e.g. (Culligan et al. 2004, Brusseau et al. 2006, Chen and Kibbey 2006, Pyrak-Nolte et al. 2008), there are some parameters that cannot be determined by standard, static experimental procedures. In this work, we present a combination of new techniques that overcome this discrepancy and allow for an estimation of the needed dynamic model parameters.

A micro-model of an idealized porous medium, i.e. a flow cell, was created from a computer aided drafting (CAD) model using stereolithography (Crandall et al. 2008). The flow cell geometry was created with an in-house computer code. The fabricated physical model geometry was known from the CAD model and thus the size of each individual interface within the flow cell could be determined by combining image analysis with the original computer model. Experiments of air invading an initially water-filled flow cell were performed. By recording images periodically during flow experiments, data on dynamic evolution of interfaces, $a_{w n}, p_{c}, S$, and velocity of interfaces were determined.

\section{NOMENCLATURE}

$a_{w n} \quad$ Specific interfacial area between wetting and non-wetting fluids $\left(\mathrm{m}^{2}\right)$

$\mathrm{Ca} \quad$ Capillary number (-)

$E_{w n} \quad$ Production rate of $\mathrm{a}_{\mathrm{wn}}\left(\mathrm{m}^{2} \mathrm{~s}^{-1}\right)$

$g \quad$ Gravitational force $(\mathrm{N})$

$h_{t} \quad$ Height of throat (m)

$I_{j} \quad$ Number of interfaces $j$

$K \quad$ Permeability $\left(\mathrm{m}^{2}\right)$

$k_{r} \quad$ Relative permeability

$p \quad$ Pressure $\left(\mathrm{Nm}^{-2}\right)$

$p_{c} \quad$ Capillary pressure $\left(\mathrm{Nm}^{-2}\right)$

$q \quad$ Mean volumetric flow rate $\left(\mathrm{m}^{2} \mathrm{~s}^{-1}\right)$

$S \quad$ Fluid saturation (-)

$t \quad$ Time (s)

$v \quad$ Fluid velocity $\left(\mathrm{ms}^{-1}\right)$

$w_{t} \quad$ Width of throat (m)

$\phi \quad$ Porosity (-) $\begin{array}{ll}\rho & \text { Fluid density }\left(\mathrm{kgm}^{-3}\right) \\ \mu & \text { Absolute viscosity (Pas) }\end{array}$

\section{Subscripts \\ $n \quad$ Non-wetting fluid \\ $w \quad$ Wetting fluid}

\section{TWO-PHASE FLOW MODEL}

Hassanizadeh and Gray (1990) have derived general balance equations for two-phase flow based on thermodynamic principles. However, it is not possible to numerically model this general system as it would require an incredible amount of computational power and because a number of the involved constitutive relationships have not been determined. Niessner and Hassanizadeh (2008) described a simplified version of the general governing equations, which represents a relatively simple extension of the standard two-phase flow model, but includes the effect of interfacial area $a_{w n}$.

Their procedure resulted in a system of three partial differential equations, one for each of the two phases and one for the fluid-fluid interface. Additional constraints included summing the wetting-phase saturation $\left(S_{w}\right)$ and non-wetting phase saturation $\left(S_{n w}\right)$ to unity, setting the $p_{c}$ as the difference between non-wetting pressure $\left(p_{n w}\right)$ and wetting-phase pressure $\left(p_{w}\right)$, and a functional relationship between $a_{w n}, p_{c}$, and $S$ was provided. This yielded the following system of equations (Niessner and Hassanizadeh 2008):

$$
\begin{aligned}
& \phi \frac{\partial S_{w}}{\partial t}+\nabla \cdot v_{w}=0 \\
& v_{w}=-K \frac{k_{r w}}{\mu_{w}}\left(\nabla p_{w}-\rho_{w} g\right) \\
& \phi \frac{\partial S_{n}}{\partial t}+\nabla \cdot v_{n}=0 \\
& v_{n}=-K \frac{k_{r n}}{\mu_{n}}\left(\nabla p_{n}-\rho_{n} g\right) \\
& \frac{\partial a_{n w}}{\partial t}+\nabla \cdot\left(a_{w n} v_{w n}\right)=E_{w n} \\
& v_{w}=-K{ }_{w n} \cdot \nabla a_{w n} \\
& S_{w}+S_{n}=1 \\
& p_{n}-p_{w}=p_{c} \\
& a_{w n}=a_{w n}\left(S_{w}, p_{c}\right)
\end{aligned}
$$

where $v$ is the Darcy velocity, $\mu$ is the dynamic viscosity, $t$ is time, $\phi$ is the porous medium porosity, $K$ is the intrinsic permeability, $\mathrm{v}_{\mathrm{wn}}$ is the interfacial velocity, and $K_{w n}$ is interfacial permeability. The $a_{w n}$ and the $E_{w n}$ are dependent on the primary variables $S_{w}$ and $p_{c}$. Relative permeabilities are only dependent on $S_{w}$ (Helmig 1997) 
To model the system given in Eqn. (1) to Eqn. (9) the $S_{w}$, $p_{c}$, and $a_{w n}$ must be determined. A functional relationship $a_{w n}\left(S_{w}, p_{c}\right)$ was used by Niessner and Hassanizadeh (2008). Ideally, a number of subsequent static drainage and imbibition experiments would be needed to obtain a reliable relationship. The variation of the $a_{w n}\left(S_{w}, p_{c}\right)$ relationship with different porous media will become more apparent as various researchers study different applications (Brusseau et al. 2006, Pyrak-Nolte et al. 2008), but currently a generic relationship from porethroat modeling suffices (Joekar-Niasar et al. 2008)

Dynamic properties that have not been determined in previous studies had to be estimated in the recent model of Niessner and Hassanizadeh (2008). These include the interfacial velocity $\mathrm{v}_{\mathrm{wn}}$, interfacial permeability $\mathrm{K}_{\mathrm{wn}}$, and the production rate of specific interfacial area $\mathrm{E}_{\mathrm{wn}}$. The remainder of this paper will review to experimental devices and techniques we used to determine these parameters and present preliminary results.

\section{EXPERIMENTAL SETUP}

The porous medium used for our experiments was constructed using stereolithography (SL) rapid prototyping, a production technique that has been used to make complex parts from computer aided design models. SL models are constructed by curing successive layers of photo-sensitive resin with a laser to form 3D objects. Our SL flow cell was constructed using a 3D Systems Viper $\mathrm{Si}^{2 \circledast}$ stereolithography apparatus out of DSM 11120 Watershed (DSM Somos, New Castle DE, USA), a water-resistant resin. The flow cell production procedure is reviewed in detail by Crandall et al. (2008).

The porous matrix of the flow cell, labeled in Figure 1, was constructed as a square-lattice of over 5000 throats within a $10.16 \mathrm{~cm}$ by $10.16 \mathrm{~cm}$ region. The individual throat widths varied from $0.35 \mathrm{~mm}$ to $1.0 \mathrm{~mm}$. These throat widths were randomly distributed throughout the porous matrix. To introduce a greater range of pore-level resistances into the flow cell, seven different throat heights were assigned to these throats, varying from $0.2 \mathrm{~mm}$ to $0.8 \mathrm{~mm}$. These throat heights were assigned in such a manner so as to reduce the aspect ratio of the throats. That is, the narrowest throats were assigned to be the shortest (smallest throat width $=$ smallest throat height), the widest throats were made the tallest, and so forth. A detailed comparison of the throat capillary and inertial resistances of this SL flow cell to resistances of other flow devices in the literature is listed in Table 1 of Crandall et al. (2008). Two manifolds were created on opposing edges of the porous matrix, arbitrarily labeled \#1 and \#2 in Figure 1. Air was injected into the centered port of one of these manifolds and through the initially water-saturated flow cell to perform the experiments.

A relatively simple experimental setup was used to control the fluid flow into the flow cell and to capture the images. A schematic of the experimental setup is shown in Figure 2 and consists of the SL flow cell, a constant-rate syringe pump (KD

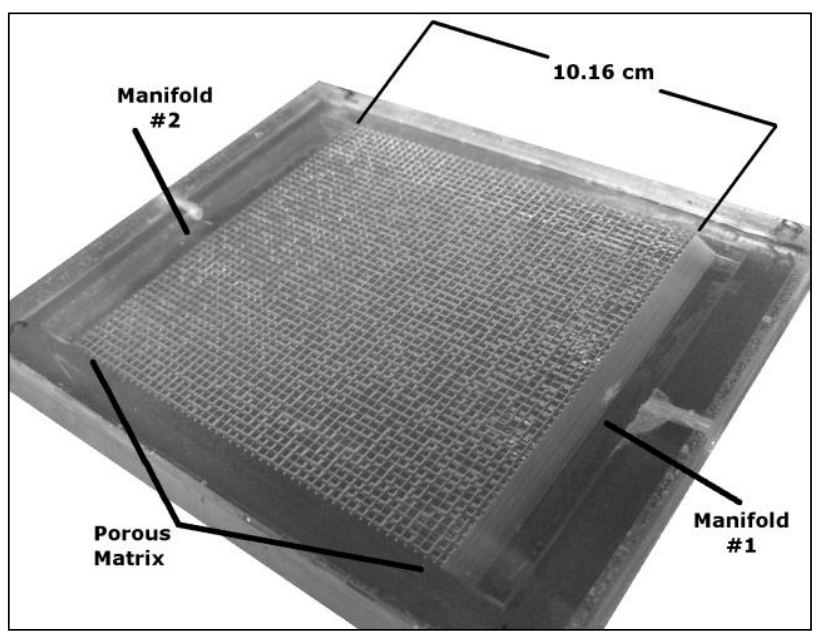

Figure 1: Photograph of stereolithography flow cell.

Scientific KDS 200), a CCD camera (NTSC COHU 4915400/000), lighting, a collection vessel at atmospheric pressure, and a data acquisition computer. A LabVIEW ${ }^{\mathrm{TM}}$ (version 7.1) module was used to control the image-capture rate. The same experimental setup was used by Crandall et al. (2009) to capture images of fluid motion in the flow cell. A postprocessing routine was written to determine the size and distribution of fluid bursts associated with Haines jumps and these were shown to be well described by the model of SelfOrganized Criticality (Crandall et al. 2009). For the current study this post-processing code was changed to identify the location and size of individual interfaces between fluids in the flow cell. By linking the known height and width of the flow cell at each location an interface was identified the fluid-fluid interface was determined. For the results presented here the individual interfaces were estimated as the cross-sectional area of the throat they were identified in. While the curvature of the interface at each location would increase the total interfacial area, for this preliminary study this approximation was deemed satisfactory.

\section{Camera}

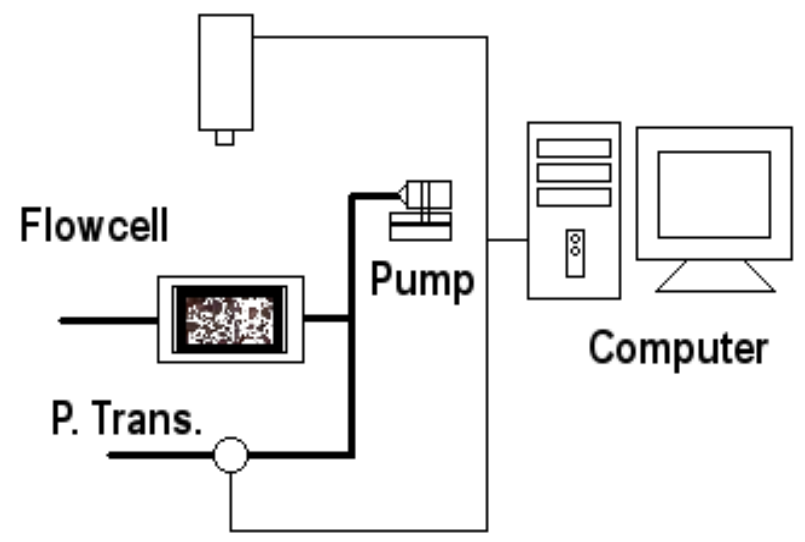

Figure 2: Schematic of experiment. 
Injection of air at different flow rates was conducted. The ratio of inertial to capillary forces for the different flow rates was quantified using the following definition of the capillary number,

$$
C a=\frac{q \mu_{w}}{\gamma}
$$

where $q$ is the mean volumetric flow across the flow cell, $\mu_{w}$ is the wetting fluid viscosity, and $\gamma$ is the interfacial tension between the injected air and the defending water $(\gamma=72 \mathrm{mN} / \mathrm{m})$. Here $q$ is defined as the injected volumetric flow rate divided by the mean cross-sectional area of the flow cell perpendicular to the flow direction. For this study the volumetric flow rate was varied from a maximum of $20 \mathrm{ml} / \min$ to a minimum of $0.002 \mathrm{ml} / \mathrm{min}$, which corresponds to $4.63\left(10^{-9}\right) \leq \mathrm{Ca} \leq$ $4.63\left(10^{-13}\right)$. All experiments were conducted with the flow cell initially saturated with distilled water and air injected into either Manifold \#1 or Manifold \#2 at a constant rate. This fluid pairing has a viscosity ratio $\left(M=\mu_{\text {air }} / \mu_{\text {water }}\right)$ of 0.0178 , which indicates the drainage case studied is in the unstable regime (Lenormand et al. 1988).

In order to determine the macro-scale parameters that are needed for the numerical model the size of an averaging volume (Representative Elemental Volume, REV) needed to be determined for the flow cell. We subdivided the flow cell in $n \times$ $n$ sub-volumes and calculated the $\phi$ of each REV as a test parameter. As the flow cell was constructed using a random distribution of throats and pores the flow cell as a whole is considered a homogeneous porous medium with a porosity of $35.79 \%$ (Crandall et al. 2008). The sub-division of the flow cell to determine the REV for which $\phi$ varies little using the largest possible value for $n$ allowed us to consider multiple regions within the porous medium as homogeneous. Table 1 shows average, maximum, minimum values of $\phi$, as well as the standard deviation for $n=2$ to 6 sized REVs. As is shown the standard deviation between the $\phi$ values were below $1 \%$ for $n=$ 2 and 3, i.e. 4 and 9 separate REVs. These lower values of variation between separate volumes were thought to be acceptable for reporting of $a_{w n}$ values, and thus the results of $E_{w n}$ are presented for each. Two images of air invasion into the flow cell are shown in Figure 3 with the $n=2$ and $n=3$ REVs identified.

\begin{tabular}{|c|c|c|c|}
\hline $\begin{array}{l}\text { Avg. } \\
\phi(\%)^{\text {Avg }}\end{array}$ & $(\%)^{\text {Min. } \phi}$ & $\begin{array}{l}\text { Max. } \\
\phi(\%)^{\text {Max. }}\end{array}$ & $(\%)^{\text {Std. Dev. }}$ \\
\hline 35.89 & 35.41 & 36.71 & 0.57 \\
\hline 35.61 & 34.23 & 37.21 & 0.92 \\
\hline 35.99 & 34.16 & 38.82 & 1.28 \\
\hline 35.77 & 32.08 & 38.87 & 1.66 \\
\hline 35.63 & 29.67 & 39.14 & 2.01 \\
\hline
\end{tabular}

Table 1: Average, minimum and maximum porosity $(\phi)$ of potential REVs within the flow cell, and the standard deviation of the values.

\section{RESULTS AND DISCUSSION}

The number of identified interfaces within the entire flow cell for an 11 hour, $0.002 \mathrm{ml} / \mathrm{min}$ flow rate experiment is shown in Figure 4 as a function of time. Also the interfaces identified in each of the four $n=2 \mathrm{REVs}$ are shown. The overall number of interfaces increases approximately linearly while the number of interfaces in each REV is observed to increase sporadically as the invading air moves in only one REV at a time at this low flow rate. This pattern is similar to the measured $S_{n w}$ of air shown in Figure 5 as a function of time. These $S$ values were determined by summing the volume of invaded flow cell regions and dividing by the total volume of the flow cell, or the volumes within the REVs.

To determine the $a_{w n}$ within the cell the interfacial area was summed and divided by the total volume of the flow cell. These values are shown in Figure 6 for the same 11 hour, $0.002 \mathrm{ml} / \mathrm{min}$ flow rate experiment in both the entire cell and within the $n=2$ REVs as a function of the $S_{n w}$. As can be seen the $a_{w n}$ increases approximately linearly for all the measured regions, with similar slopes both in the entire cell and in each REV.
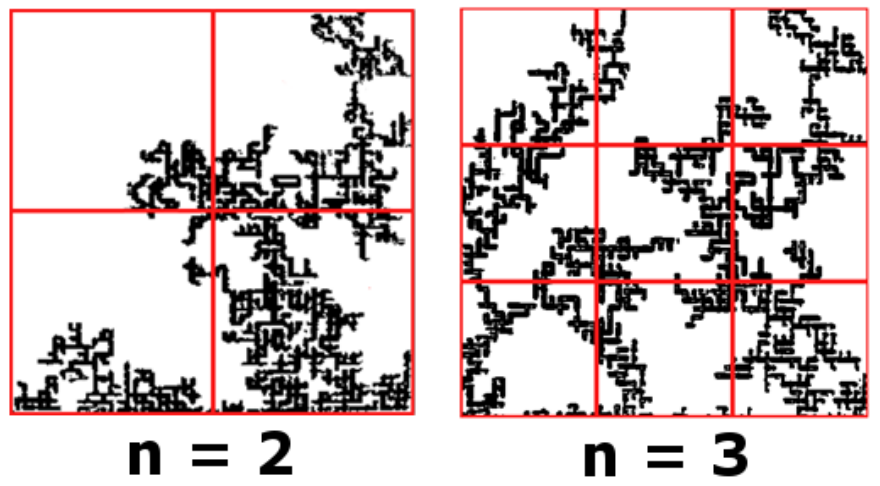

Figure 3: REVs for $n=2$ and $n=3$ shown on top of experimental images of air in the water filled flow cell.

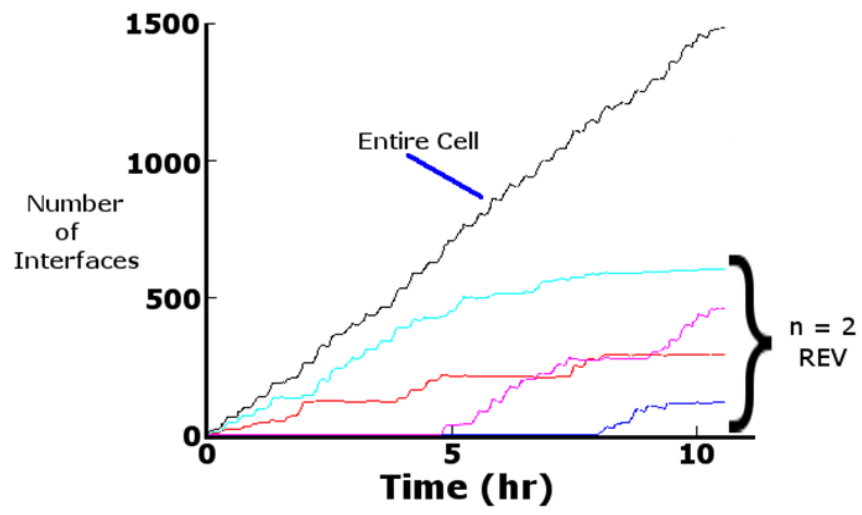

Figure 4: Identified interfaces in the entire flow cell and in each of the $n=2$ REVs. 


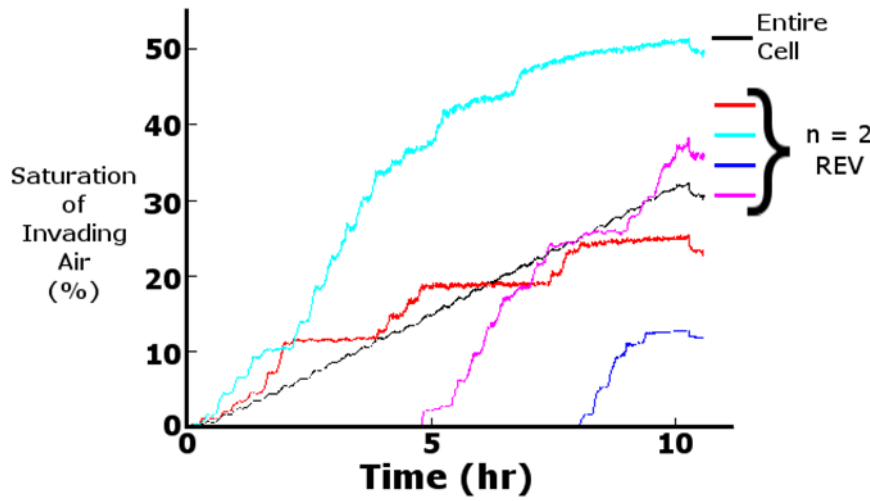

Figure 5: Saturation of air in the entire flow cell and in each of the $n=2 \mathrm{REVs}$.

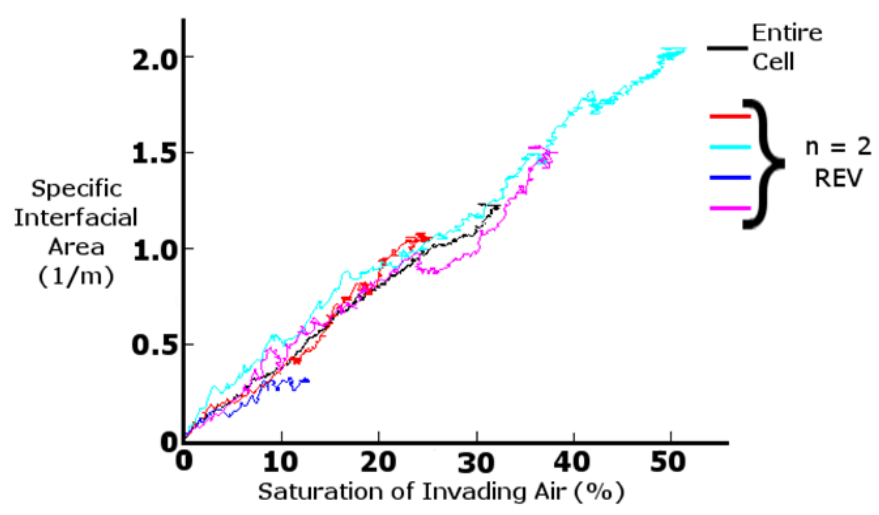

Figure 6: $a_{w n}$ in each of the $n=2$ REVs and the flow cell.

The pressure across the entire flow cell was measured with a pressure transducer, as shown in the experimental schematic in Figure 2. These pressure values are shown in Figure 7 for the same 11 hour, $0.002 \mathrm{ml} / \mathrm{min}$ flow rate experiment shown in Figures 4 - 6. The pressure increased gradually to approximately $675 \mathrm{~Pa}$, with fluctuations of about $100 \mathrm{~Pa}$ every 15 minutes. To estimate the average $p_{c}$ of the interfaces the individual $p_{c}$ at each throat was determined,

$p_{c}=\frac{2 \gamma}{h_{t}+w_{t}}$

where $h_{t}$ is the height of the throat the interface was measured in and $w_{t}$ is the width. The average $p_{c}$ was determined by summing the individual $p_{c}$ and dividing by the total number of interfaces measured. This average $p_{c}$ is shown in Figure 8 for the entire cell and within each of the $n=3$ REVs. The average $p_{c}$ value also fluctuates significantly over the experiment and is within the same range of values as the macroscopically measured pressure difference across the cell.

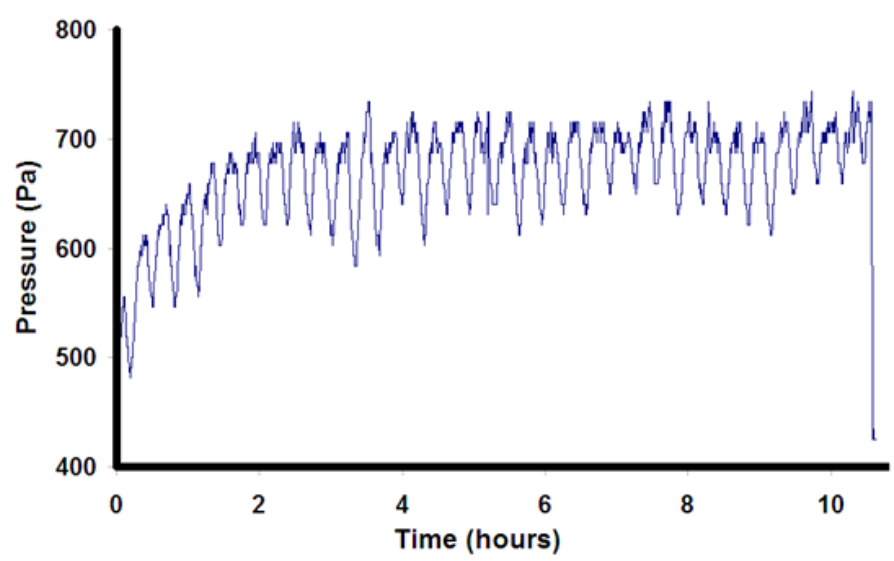

Figure 7: Pressure measured across the flow cell.

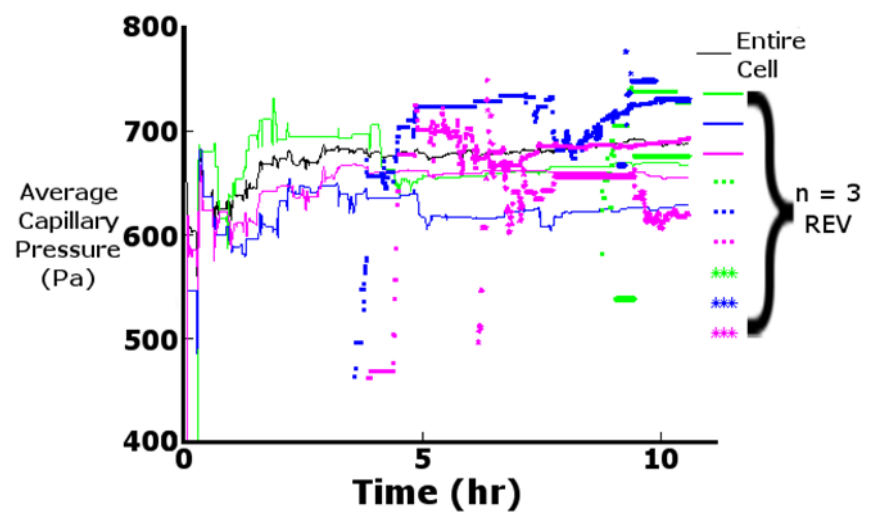

Figure 8: $a_{w n}$ in each of the $n=3$ REVs and the entire cell.

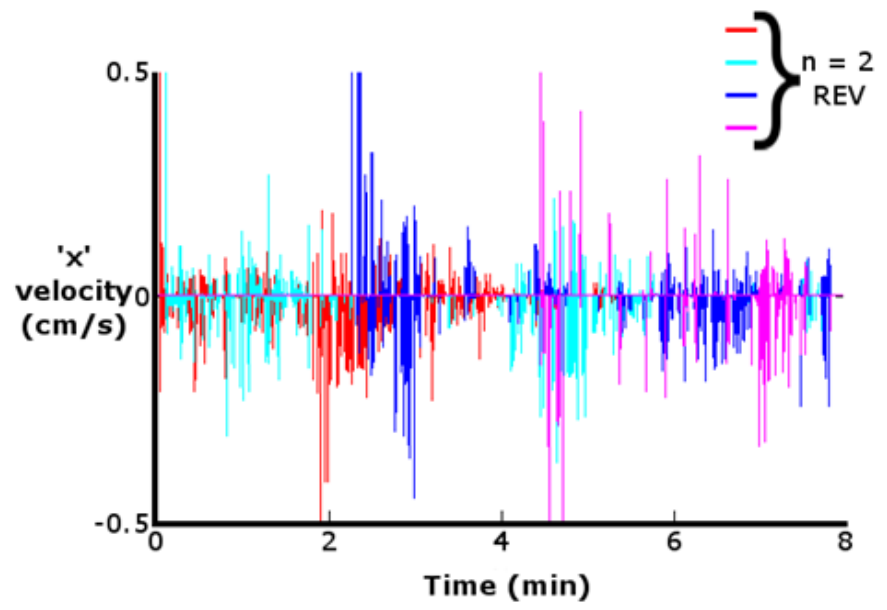

Figure 9: $v_{w n}$ in each of the $n=2 \mathrm{REVs}$ the $\mathrm{x}$ direction. 


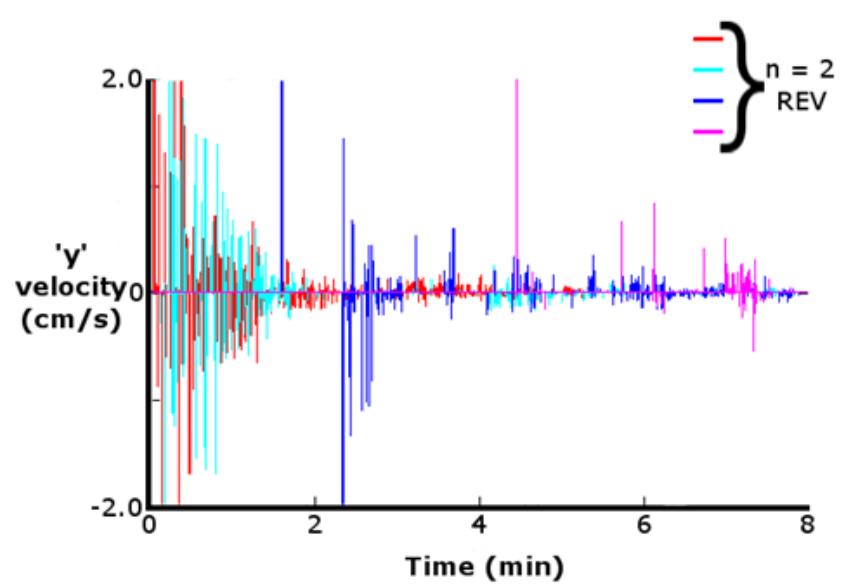

Figure 10: $v_{w n}$ in each of the $n=2$ REVs the y direction.

Our preliminary measurements of the interfacial velocity $\left(v_{w n}\right)$ reveal a complex and sporadic pattern of interfacial motion. The $v_{w n}$ was determined for both the $\mathrm{x}$ and the $\mathrm{y}$ directions, where $\mathrm{y}$ is in the bulk flow direction. By averaging the number of interfaces that moved a distance in the $\mathrm{x}\left(I_{x}\right)$ and $\mathrm{y}\left(I_{y}\right)$ directions in between successive images the $v_{w n}$ was determined for both the $\mathrm{x}$ and the $\mathrm{y}$ direction.

$$
\begin{aligned}
& \left(v_{w n}\right)_{x}=\frac{1}{I_{x}} \sum_{i=1}^{I_{x}} \frac{\Delta x}{\Delta t} \\
& \left(v_{w n}\right)_{y}=\frac{1}{I_{y}} \sum_{i=1}^{I_{y}} \frac{\Delta y}{\Delta t}
\end{aligned}
$$

As shown in Figures 9 and 10 this resulted in a chaotic pattern of velocity values in both positive and negative directions. This jumpy pattern is due to the occurrence of Haines jumps as the non-wetting air quickly invades water filled pores (Crandall et al. 2009). By averaging over a reasonable amount of time we are hoping to see a smoother description of the $v_{w n}$ emerge from these initial results. The overall average of the $v_{w n}$ in the $x$ direction is approximately zero, as would be expected since the bulk fluid motion is in the $y$ direction. Once we have a reasonable set of velocity measurements we will determine the $K_{w n}$ using the following relation,

$$
K_{j j, w n}=-v_{j, w n} / \frac{\partial a_{w n}}{\partial j}
$$

and $E_{w n}$ will be determined via Eqn. 5. Both of these dynamic quantities rely on an accurate description of the $v_{w n}$ on a macroscopic scale.

\section{CONCLUSIONS}

We have performed experiments of air flowing within a stereolithography flow cell. Images of air invasion were captured during the experiments. With knowledge of the porous medium geometry the location and size of the interfaces within the flowing air structure was determined. The number of interfaces was observed to increase in a linear fashion during the primary drainage flows evaluated for this paper. The capillary pressure determined from the image analysis technique was shown to be in good agreement with the pressure measured across the flow cell via a pressure transducer. Initial measurements of the interfacial velocity show a great deal of scatter due to the sporadic motion of the interface at the pore level. This is because of the small capillary resistances being overcome as the non-wetting fluid displaces the water. By averaging these velocity values we hope to be able to determine interfacial production and permeability values for our experiments.

\section{ACKNOWLEDGMENTS}

This material is declared a work of the U.S. Government and is not subject to copyright protection in the United States. Approved for public release; distribution is unlimited.

The authors extend their gratitude to Jen Niessner and Majid Hassanizadeh for the impetus to study this topic and conversations on the matter. Also we wish to thank Martin Ferer for his knowledgeable talks on flow in porous media.

\section{REFERENCES}

Brusseau, M.L., S. Peng, G. Schnaar, and M.S. CostanzaRobinson. 2006. Relationships among air-water interfacial area, capillary pressure, and water saturation for a sandy porous medium. Water Resour. Res. 42:W03501.

Chen, L., and T.C.G. Kibbey. 2006. Measurement of airwater interfacial area for multiple hysteretic drainage curves in an unsaturated fine sand. Langmuir 22:6674-6880.

Crandall, D., G. Ahmadi, M. Ferer, and D.H. Smith. 2009. Distribution and occurrence of localized-bursts in two-phase flow through porous media. Physica A 38:574-584.

Crandall, D., G. Ahmadi, D. Leonard, M. Ferer, and D.H. Smith. 2008. A new stereolithography experimental porous flow device. Rev. Sci. Instrum. 79(4):044501.

Culligan, K.A., D. Wildenschild, B.S.B. Christensen, W. G. Gray and M.L. Rivers. 2006. Pore-scale characteristics of multiphase flow in porous media: A comparison of air-water and oil-water experiments. Adv. Water Resour. 29:227-238.

Darcy, H. 1856. Les fontaines publiques de la ville de Dijon. Dalmont, Paris.

Hassanizadeh, S.M. and W.G. Gray. 1980. General conservation equations for multiphase systems: 3 . Constitutive theory for porous media flow. Adv. Water Resour. 13(4):169186.

Hassanizadeh, S.M. and W.G. Gray. 1990. Mechanics and thermodynamics of multiphase flow in porous media including interphase boundaries. Adv. Water Res. 13(4):169-186.

Gray, W.G. and C.T. Miller. 2005.Thermodynamically constrained averaging theory approach for modeling of flow in 
porous media: 1 Motivation and overview. Adv. Water Resour. 28(2): 161180 .

Gray, M.L. Rivers and A.F.B. Tompson. 2004. Interfacial area measurements for unsaturated flow through a porous medium. Water Resour. Res. 40(12):1-12.

Helmig, R. 1997. Multiphase Flow and Transport Processes in the Subsurface. Springer, Berlin.

Hilfer, R. and F. Doster. 2009. Percolation as a basic concept for macroscopic capillarity. Trans. Porous Media.

Joekar-Niasar, V., S.M. Hassanizadeh, and A. Leijnse. 2008. Insights into the relationship among capillary pressure, saturation, interfacial area and relative permeability using porescale network modeling. Trans. Porous Media 74(2):201-219.

Lenormand, R., E. Touboul, and C. Zarcone. 1988. Numerical models and experiments on immiscible displacements in porous media. J. Fluid Mech. 189:165-187.

Miller, C.T. and W.G. Gray. 2005.Thermodynamically constrained averaging theory approach for modeling of flow in porous media: 2. Foundation. Adv. Water Resour. 28(2): 181202.

Niessner, J., and S.M. Hassanizadeh. 2008. A model for two-phase flow in porous media including fluid-fluid interfacial area. Water Resour. Res. 44, W08439.

Pyrak-Nolte, L.J., D.D. Nolte, D.Q. Chen, and N.J. Giordano. 2008. Relating capillary pressure to interfacial areas. Water Resour. Res. 44:W06408. 